\title{
Tacrolimus Toxicity Associated with Concomitant Metoclopramide Therapy
}

\author{
William A. Prescott, Jr., Pharm.D., Brian L. Callahan, Pharm.D., \\ and Jeong M. Park, Pharm.D., M.S.
}

\begin{abstract}
Subtherapeutic tacrolimus trough concentrations were noted in a 52-year-old woman who had undergone liver transplantation. Her tacrolimus dosage was increased from 7 to $28 \mathrm{mg}$ twice/day, and ketoconazole therapy was added; however, her tacrolimus concentration remained undetectable. Metoclopramide $10 \mathrm{mg} 4$ times/day was begun to control the patient's newonset nausea and vomiting. Within 48 hours of increasing the dosage to 20 mg 4 times/day, her tacrolimus trough concentration exceeded $30 \mathrm{ng} / \mathrm{ml}$. Signs and symptoms were suggestive of tacrolimus nephrotoxicity and neurotoxicity. According to the Naranjo scale, this adverse drug event was probably the result of improved absorption of tacrolimus secondary to metoclopramide therapy. The patient's subtherapeutic tacrolimus concentration at baseline was probably secondary to poor absorption due to impaired gastric emptying. Coadministration of metoclopramide significantly improved gastric motility and delivery of tacrolimus to the small intestine, increasing tacrolimus bioavailability, thus resulting in acute-onset tacrolimus toxicity. When tacrolimus is administered with metoclopramide in patients with gastric dysmotility, tacrolimus concentrations should be monitored closely to minimize the risk of toxicity.
\end{abstract}

Key Words: tacrolimus, metoclopramide, drug interaction, gastric motility.

(Pharmacotherapy 2004;24(4):532-537)

Tacrolimus, a macrolide with potent immunosuppressive properties, is approved by the Food and Drug Administration for prophylaxis against rejection after liver and kidney transplantation. Tacrolimus absorption, like cyclosporine absorption, is variable, incomplete, and dependent on proper gastric motility. ${ }^{1,2}$ Metoclopramide, a prokinetic agent commonly administered to treat gastric dysmotility, accelerates gastric emptying and intestinal transit. ${ }^{3}$ The manufacturer of tacrolimus reports that due to effects on gastric motility, metoclopramide may increase the bioavailability of tacrolimus. ${ }^{1}$ Concomitant administration of these two agents thus may

From the Department of Pharmacy Services, College of Pharmacy, University of Michigan Health System, Ann Arbor, Michigan (all authors).

Address reprint requests to Jeong M. Park, M.S., Pharm.D., University of Michigan Health System, Department of Pharmacy Services-UH B2D301, 1500 East Medical Center Drive, Ann Arbor, MI 48109-0008. increase whole blood tacrolimus concentration and the risk of toxicity. However, documentation of such an interaction between metoclopramide and tacrolimus remains poor. We describe the first case report, to our knowledge, of a clinically significant pharmacodynamic interaction between these two drugs.

\section{Case Report}

A 52-year-old, 91-kg woman with a history of end-stage liver disease secondary to primary biliary cirrhosis underwent liver transplantation approximately 11.5 years ago. About 7 years later, a liver biopsy showed chronic rejection, and the patient's therapy was changed from cyclosporine to tacrolimus. The patient received tacrolimus and prednisone for maintenance therapy for about 3 years; at that time, sirolimus was added to her immunosuppressant regimen. 
Table 1. Correlation Between Concomitant Drugs Administered with Tacrolimus and Whole Blood Tacrolimus Concentrations

\begin{tabular}{|c|c|c|c|}
\hline Date & $\begin{array}{c}\text { Tacrolimus } \\
\text { Concentration } \\
(\mathrm{ng} / \mathrm{ml})\end{array}$ & $\begin{array}{l}\text { Tacrolimus } \\
\text { Dosage }\end{array}$ & Concomitant Drugs Administered \\
\hline $11-18-02$ & $<2.0$ & $7 \mathrm{mg}$ b.i.d. & Sirolimus 4 mg q.d. \\
\hline $12-2-02$ & $<2.0$ & $14 \mathrm{mg}$ b.i.d. & \\
\hline $12-10-02$ & $<2.0$ & & \\
\hline $12-14-02$ & $<2.0$ & & \\
\hline $12-15-02$ & & & Sirolimus increased to $7.5 \mathrm{mg}$ b.i.d. \\
\hline $12-17-02$ & $<2.0$ & $28 \mathrm{mg}$ b.i.d. & \\
\hline $12-18-02$ & $<2.0$ & & Rifampin 300 mg b.i.d. started \\
\hline $12-20-02$ & $<2.0$ & & \\
\hline $12-21-02$ & $<2.0$ & & Rifampin discontinued. \\
\hline $12-22-02$ & $<2.0$ & & Cimetidine $400 \mathrm{mg}$ q.d. started. \\
\hline $12-23-02$ & $<2.0$ & & $\begin{array}{l}\text { Cimetidine discontinued, } \\
\text { ketoconazole } 200 \text { mg q.d. started. }\end{array}$ \\
\hline $12-24-02$ & $<2.0$ & & \\
\hline $12-27-02$ & $<2.0$ & & Metoclopramide $10 \mathrm{mg}$ q.i.d. started. \\
\hline $12-30-02$ & $<2.0$ & & \\
\hline $12-31-02$ & $<2.0$ & & Metoclopramide increased to $20 \mathrm{mg}$ q.i.d. \\
\hline $1-01-03$ & 5.6 & & \\
\hline $1-02-03$ & $>30$ & $20 \mathrm{mg}$ b.i.d. & \\
\hline $1-04-03$ & $>30$ & Discontinued & \\
\hline $1-05-02$ & $>30$ & & Sirolimus and ketoconazole discontinued. \\
\hline $1-06-03$ & 24.9 & & \\
\hline $1-07-03$ & 14.3 & 4 mg b.i.d. & \\
\hline $1-11-03$ & 10.5 & & Metoclopramide $10 \mathrm{mg}$ b.i.d. restarted. \\
\hline $1-24-03$ & 10.5 & $\begin{array}{l}4 \mathrm{mg} \text { in A.M. } \\
3 \mathrm{mg} \text { in P.M. }\end{array}$ & \\
\hline $2-26-03$ & & & Metoclopramide discontinued. \\
\hline $3-04-03$ & 11.8 & & \\
\hline $5-07-03$ & 12.4 & $3 \mathrm{mg}$ b.i.d. & \\
\hline $5-16-03$ & 8.9 & $2 \mathrm{mg}$ b.i.d. & \\
\hline $5-17-03$ & 6.8 & $1 \mathrm{mg}$ b.i.d. & \\
\hline
\end{tabular}

Her tacrolimus trough concentrations generally remained within the therapeutic goal range (5-10 $\mathrm{ng} / \mathrm{ml}$ ), ranging from $4.2-8.9 \mathrm{ng} / \mathrm{ml}$ in the 2 months preceding the onset of subtherapeutic tacrolimus concentrations.

About 6 months later, a tacrolimus trough level obtained on an outpatient basis revealed a concentration less than $2 \mathrm{ng} / \mathrm{ml}$, which was below the lower limit of detection of our assay (Table 1). At that time she was taking ursodiol $600 \mathrm{mg}$ twice/day, pantoprazole $40 \mathrm{mg}$ once/day, and alendronate $70 \mathrm{mg}$ once/week in addition to her immunosuppressant regimen, which consisted of prednisone $15 \mathrm{mg}$ once/day, tacrolimus $7 \mathrm{mg}$ twice/day, and sirolimus $4 \mathrm{mg}$ once/day. Of note, she had been receiving the same dosage of tacrolimus and sirolimus for at least 2 months preceding the onset of subtherapeutic tacrolimus levels.

Two weeks after the patient's tacrolimus trough level was obtained, her dosage was increased to $14 \mathrm{mg}$ twice/day. Despite this significant increase, her tacrolimus trough concentration remained undetectable. She was admitted to the hospital 10 days later with a tacrolimus trough level below $2 \mathrm{ng} / \mathrm{ml}$ and a 1 -week history of increased jaundice, itching, and thirst, which are symptoms suggestive of acute graft rejection. Her alanine aminotransferase (ALT) and aspartate aminotransferase (AST) levels on admission were 240 and 228 IU/L, respectively, elevated from baseline levels of 100-200 IU/L (normal ranges 0-45 IU/L [ALT], 2-35 IU/L [AST]). She reported dry heaves, but her appetite was unchanged. On the day of her admission, a 3-day course of intravenous methylprednisolone $60 \mathrm{mg}$ followed by a prednisone taper was added to treat the rejection empirically. On day 5, her subtherapeutic drug concentrations persisted, and her tacrolimus dosage was increased to $28 \mathrm{mg}$ twice/day.

On day 6, rifampin $300 \mathrm{mg}$ twice/day was added to control the patient's pruritic symptoms. After a pharmacy consultation on day 9, rifampin was discontinued. Because rifampin induces cytochrome P450 (CYP) 3A4, it may have been 
hindering efforts to attain a therapeutic tacrolimus concentration. Oral cimetidine 400 $\mathrm{mg}$ once/day was added on day 10 for its CYP3A4-inhibiting properties in an attempt to increase the patient's tacrolimus blood concentration. The next day, cimetidine was discontinued in favor of ketoconazole $200 \mathrm{mg}$ once/day, as ketoconazole may be a more potent inhibitor of CYP3A4.

Inadequate immunosuppression was a concern; thus, mycophenolate mofetil was added to her regimen on day 11 before an adequate trial with ketoconazole. On day 14, the patient had a lowgrade fever and complained of new-onset nausea and vomiting with any oral intake. To control her symptoms, metoclopramide $10 \mathrm{mg} 4$ times/day was started. An esophagogastroduodenoscopy conducted on day 18 did not reveal any physiologic cause for her nausea and vomiting. Ondansetron $4 \mathrm{mg} 4$ times/day was added, and her metoclopramide dosage was increased to $20 \mathrm{mg} 4$ times/day on day 19. After this her nausea and vomiting improved.

During this hospital stay the patient was also treated with ursodiol, levofloxacin, metronidazole, ranitidine, omeprazole, and pantoprazole, none of which seemed to have a definite effect on her tacrolimus concentrations. She was discharged to home on day 20, with a regimen of tacrolimus $28 \mathrm{mg}$ twice/day, sirolimus $7.5 \mathrm{mg}$ twice/day, mycophenolate mofetil $500 \mathrm{mg}$ twice/day, prednisone $60 \mathrm{mg}$ once/day, ursodiol $600 \mathrm{mg}$ twice/day, ketoconazole $200 \mathrm{mg}$ once/day, omeprazole $20 \mathrm{mg}$ twice/day, ondansetron $4 \mathrm{mg}$ every 8 hours, and metoclopramide $20 \mathrm{mg}$ before meals and at bedtime.

On the day of the patient's discharge, her tacrolimus trough concentration was $5.6 \mathrm{ng} / \mathrm{ml}$. A tacrolimus trough concentration obtained on an outpatient basis the next day revealed a supratherapeutic level greater than $30 \mathrm{ng} / \mathrm{ml}$, which is the upper limit of detection for our assay. The patient's tacrolimus dosage was decreased to $20 \mathrm{mg}$ twice/day. Two days later, she came to the emergency department with complaints of tremor, weakness, headache, leg pain, and nausea and vomiting. She also reported a 2-day history of diarrhea, which had worsened progressively.

Elevated blood urea nitrogen and serum creatinine levels of 58 and $3.3 \mathrm{mg} / \mathrm{dl}$, respectively, from baseline values of 20 and $1.1 \mathrm{mg} / \mathrm{dl}$, respectively, indicated that the patient was experiencing acute renal failure. Fractional excretion of sodium was equal to $3.79 \%$ (normal
$<2.0 \%$ ), suggesting acute tubular necrosis, which may have been indirectly related to tacrolimus toxicity. Her ALT and AST levels, although elevated at 190 and 144 IU/L, respectively, were not significantly increased from baseline. Ketoconazole was discontinued on readmission to the hospital, and tacrolimus was temporarily withheld.

On day 2 after the patient's readmission, sirolimus and mycophenolate mofetil were discontinued given the high degree of immunosuppression she experienced with supratherapeutic tacrolimus levels. Metoclopramide, ondansetron, and pantoprazole were continued throughout this hospital stay. Tacrolimus levels greater than $30 \mathrm{ng} / \mathrm{ml}$ persisted for 2 days, when they began to trend downward. The patient's renal function improved as her tacrolimus concentration decreased. Liver function tests remained stable throughout her 4-day hospital stay.

On day 4, tacrolimus $4 \mathrm{mg}$ twice/day was restarted; the goal was to maintain blood levels of 5-10 ng/ml. Seventeen days later, tacrolimus was decreased from $4 \mathrm{mg}$ twice/day to $4 \mathrm{mg}$ in the morning and $3 \mathrm{mg}$ in the evening. About 2 weeks earlier, metoclopramide had been decreased to $10 \mathrm{mg}$ twice/day; about 4 weeks after that, metoclopramide therapy was discontinued due to concern that it may be contributing to her hand tremor.

About 4 months later, the patient was receiving tacrolimus $1 \mathrm{mg}$ twice/day, and her whole blood trough concentration was stable at $6.8 \mathrm{ng} / \mathrm{ml}$. This dosage was $14 \%$ of the original dosage (tacrolimus $7 \mathrm{mg}$ twice/day) that she had taken for maintenance during the months preceding the initial hospitalization. The reason she could maintain a therapeutic tacrolimus concentration with the lower dosage remains undetermined.

\section{Discussion}

Our patient was treated for suspected gastric dysmotility with metoclopramide, which resulted in a significant increase in tacrolimus blood concentration and acute-onset tacrolimus toxicity. A Naranjo score of 5 was calculated, ${ }^{4}$ suggesting that our patient's tacrolimus toxicity was secondary to a probable pharmacodynamic interaction between metoclopramide and tacrolimus.

The significant interindividual and intraindividual pharmacokinetic and pharmacodynamic variability, along with the narrow therapeutic index of tacrolimus, necessitates routine monitoring of 
whole blood drug concentrations. Therapeutic goal trough concentrations generally range from $5-20 \mathrm{ng} / \mathrm{ml}$. The specific target concentration may vary depending on time since transplantation and on the protocol of the transplant center.

For our patient, who had undergone liver transplantation more than 10 years ago, the target tacrolimus trough concentration was 5-10 $\mathrm{ng} / \mathrm{ml}$ according to the transplant center protocol. Subtherapeutic concentrations may ultimately result in graft rejection, whereas supratherapeutic concentrations have been associated with tacrolimus toxicity. ${ }^{5-10}$ Both inpatient and outpatient tacrolimus levels in our patient were performed at the hospital by means of microparticle enzyme immunoassay, which has a detection range of $2-30 \mathrm{ng} / \mathrm{ml}$. All inpatient tacrolimus concentrations were drawn as trough levels according to hospital procedure. Whether outpatient tacrolimus concentrations were drawn as trough levels could not be definitively confirmed.

Nephrotoxicity and neurotoxicity are common adverse events seen with elevated tacrolimus concentrations. 5, 7,8,10 Generally, the symptoms associated with elevated tacrolimus levels are reversible if the tacrolimus regimen is appropriately modified. ${ }^{6}$ Our patient had complained of headache, tremor, and weakness, which are common manifestations of tacrolimusassociated neurotoxicity. Elevated blood urea nitrogen and serum creatinine levels indicated that the patient was experiencing acute renal failure. Other potential causes were ruled out, suggesting that the patient's renal dysfunction was due to acute tubular necrosis, which may have been precipitated by her high tacrolimus concentration.

Since CYP3A4 is the primary isoenzyme responsible for tacrolimus metabolism, clinically relevant drug interactions can occur when agents that inhibit or induce this isoenzyme are administered concomitantly. Inhibition of tacrolimus metabolism by agents such as ketoconazole may result in an increased risk for nephrotoxicity and neurotoxicity. ${ }^{11-14}$ Induction of CYP3A4 by agents such as rifampin leads to reduced tacrolimus bioavailability and a higher rate of clearance, ultimately resulting in subtherapeutic levels and impaired efficacy. ${ }^{14-18}$

Although the onset of enzyme inhibition is rapid, enzyme induction is a slow, timedependent process. ${ }^{11}$ The onset of clinically significant elevations in whole blood tacrolimus concentrations in ketoconazole-treated patients may be noted as soon as 1 day after the start of therapy, whereas rifampin may not exert a clinically significant effect on concentration until 2-12 days of therapy. ${ }^{14,16,18}$

The subtherapeutic tacrolimus trough concentrations in our patient probably were not the result of extensive intrinsic metabolic clearance. If enhanced metabolism had been the cause, the addition of ketoconazole, which would have rapidly caused CYP3A4 inhibition, would have resulted in a prompt increase in tacrolimus concentration. However, the patient's tacrolimus levels remained subtherapeutic for 10 days after the start of ketoconazole therapy. It seems unlikely that this treatment resulted in a delayedonset inhibition of CYP3A4. Metoclopramide inhibits CYP2D6 but has no known effect on CYP3A4; therefore, a drug interaction with tacrolimus is unlikely to occur by way of this mechanism. ${ }^{3}$

Rifampin was added to the patient's drug regimen on day 6 after her hospital admission even though this agent lowers whole blood tacrolimus concentrations. ${ }^{14-18}$ Thus, the rifampin might have induced the CYP3A4 enzyme system, potentially impairing the ability of ketoconazole to inhibit this isoenzyme adequately and increase tacrolimus levels. However, one published case report suggested that after discontinuing rifampin after 4 days of therapy, enzyme induction and accelerated tacrolimus metabolism dissipated within 6 days. ${ }^{14}$

Our patient was treated with rifampin for only 3 days; rifampin was discontinued 2 days before ketoconazole therapy was begun, and no increase in tacrolimus levels occurred for 9 additional days. Thus, it is unlikely that rifampin had any clinically significant effect on impairing or delaying the ability of ketoconazole to exert its inhibition of CYP3A4. If it had, given the rapid nature of ketoconazole enzyme inhibition, the patient's tacrolimus concentration would likely have increased much sooner after rifampin discontinuation. Thus, it is also unlikely that ketoconazole itself was the underlying cause of the patient's tacrolimus toxicity because, as mentioned, the effect on tacrolimus would have been observed much earlier than it was. ${ }^{14}$

Sirolimus at doses of $2 \mathrm{mg}$ and above has been shown to reduce the area under the concentrationtime curve (AUC) of tacrolimus when administered concomitantly. ${ }^{19}$ Tacrolimus and sirolimus, in stable doses, were administered concomitantly for several months before the onset of the subtherapeutic tacrolimus concentrations, and 
therapy with sirolimus was continued through the onset of supratherapeutic tacrolimus levels. A similar effect of sirolimus on tacrolimus concentrations was not observed in our patient; thus, the changes in tacrolimus concentrations probably were not due to an interaction with sirolimus.

The bioavailability of orally administered tacrolimus is generally poor and erratic, averaging about $25 \%$ and ranging from $4-89 \%{ }^{3}$ This is likely due to extensive first-pass metabolism in the intestine and liver as well as variability in the intestinal and hepatic contents of CYP3A4 and P-glycoprotein. ${ }^{20,21}$ In addition, the rate and extent of tacrolimus absorption have decreased when tacrolimus was administered with food, especially a meal high in fat, by delaying gastric emptying. ${ }^{22,}{ }^{23}$ The impaired tacrolimus absorption seen in certain patient populations thus may be the result of alterations in gastric motility. ${ }^{5}$ This was speculated to be the underlying etiology of our patient's persistent subtherapeutic tacrolimus concentrations.

Cyclosporine, like tacrolimus, is absorbed predominantly in the small intestine. Absorption of these immunosuppressants is thus dependent on proper gastric emptying. One study assessed the effect of coadministered oral metoclopramide on the absorption of oral cyclosporine 4-10 $\mathrm{mg} / \mathrm{kg} /$ day (mean $7.5 \mathrm{mg} / \mathrm{kg} /$ day) in 14 patients who underwent kidney transplantation. ${ }^{2}$ When metoclopramide was added to the cyclosporine regimen, the area under the concentration-time curve of cyclosporine increased by $29 \%$ $(\mathrm{p}=0.003)$, and cyclosporine peak concentration increased in 22 of 24 patients studied ( $\mathrm{p}=0.00005)$.

In addition, the time to reach peak concentration significantly decreased with concurrent metoclopramide therapy $(\mathrm{p}=0.01)$, indicating an increase in the rate of cyclosporine absorption. The authors concluded that the addition of metoclopramide significantly increased the rate and extent of cyclosporine absorption, and that this effect was the result of enhanced gastric emptying of cyclosporine.

Our patient's subtherapeutic tacrolimus concentrations apparently were secondary to poor absorption due to impaired gastric emptying. As an outpatient, her compliance with her drug regimen was unknown but was thought to be acceptable. In addition, given that her tacrolimus levels remained subtherapeutic throughout her prolonged hospital stay, poor compliance does not appear to have been a contributing factor. The large tacrolimus dosage she received would have been expected to produce supratherapeutic concentrations if her gastrointestinal tract was working properly, especially in the presence of a potent enzyme inhibitor such as ketoconazole.

The patient's tacrolimus trough concentration began to trend upward 5 days after metoclopramide therapy was started and 1 day after the dosage was increased from 10 to $20 \mathrm{mg} 4$ times/day. The lower dosage of metoclopramide given initially did not appear to improve the patient's gastrointestinal symptoms, whereas the higher dosage was effective. Thus, it is not surprising that the tacrolimus concentration did not improve until the metoclopramide dosage was increased. After that, the patient's tacrolimus concentration increased, an effect believed to have resulted from optimal gastric emptying.

Ketoconazole may have significantly impaired the first-pass metabolism of tacrolimus once the drug was delivered properly to its absorption site, further contributing to our patient's supratherapeutic tacrolimus concentrations. Diarrhea also causes elevations in tacrolimus levels. ${ }^{24,25}$ However, our patient's diarrhea seemed to occur after her tacrolimus levels became elevated and was thus more likely an adverse event secondary to the high levels than a cause of the toxicity. Her diarrhea, however, may have further elevated her already toxic tacrolimus concentration.

Although the dramatic changes in our patient's whole blood tacrolimus concentration could be confounded by several variables, the primary cause of her elevated levels appears to have been metoclopramide. One could argue that since removing metoclopramide from her drug regimen approximately 2 months after it was started did not result in recurrence of subtherapeutic tacrolimus levels, metoclopramide might not have been the causative agent. When it was discontinued, however, an indication for therapy no longer existed. Hence, the patient's earlier gastric dysmotility had improved and no longer impaired absorption of tacrolimus.

\section{Conclusion}

Coadministration of metoclopramide with tacrolimus in our patient resulted in significantly elevated whole blood tacrolimus concentration, probably because this therapy improved the patient's gastric motility. When metoclopramide is added to tacrolimus therapy in patients with gastric dysmotility, tacrolimus concentrations should be monitored closely to avoid tacrolimus toxicity. 


\section{References}

1. Fujisawa Healthcare Inc. Prograf (tacrolimus) prescribing information. Deerfield, IL; 2002.

2. Wadhwa NK, Schroeder TJ, O'Flaherty E, Pesce AJ, Myre SA, First MR. The effect of oral metoclopramide on the absorption of cyclosporine. Transplantation 1987;43:211-13.

3. A. H. Robins Co. Reglan (metoclopramide) prescribing information. Richmond, VA; 2001.

4. Naranjo CA, Busto U, Sellers EM, et al. A method for estimating the probability of adverse drug reactions. Clin Pharmacol Ther 1981;30:239-45.

5. Venkataramanan R, Swaminathan A, Prasad T, et al. Clinical pharmacokinetics of tacrolimus. Clin Pharmacokinet 1995;29: 404-30.

6. Kelly PA, Burckart GJ, Venkataramanan R. Tacrolimus: A new immunosuppressive agent. Am J Health-Syst Pharm 1995;52: 1521-35.

7. Winkler M, Ringe B, Baumann J, Loss M, Wonigeit K, Pichlmayr R. Plasma vs whole blood for therapeutic drug monitoring of patients receiving FK506 for immunosuppression. Clin Chem 1994;40:2247-53.

8. Backman L, Nicar M, Levy M, et al. FK506 trough levels in whole blood and plasma in liver transplant recipients. Transplantation 1994;57:519-25.

9. Venkataramanan R, Shaw LM, Sarkozi L, et al. Clinical utility of monitoring tacrolimus blood concentrations in liver transplant patients. J Clin Pharmacol 2001;41:542-51.

10. Winkler M, Jost U, Ringe B, Gubernatis G, Wonigeit K, Pichlmayr R. Association of elevated FK 506 plasma levels with nephrotoxicity in liver-grafted patients. Transplant Proc 1991;23:3153-5.

11. Christians U, Jacobsen W, Benet LZ, Lampen A. Mechanisms of clinically relevant drug interactions associated with tacrolimus. Clin Pharmacokinet 2002;41:813-51.

12. Prasad TN, Stiff DD, Subbotina N, et al. FK 506 (tacrolimus) metabolism by rat liver microsomes and its inhibition by other drugs. Res Commun Chem Pathol Pharmacol 1994:84:35-46.

13. Floren LC, Bekersky I, Benet LZ, et al. Tacrolimus oral bioavailability doubles with coadministration of ketoconazole.
Clin Pharmacol Ther 1997;62:41-9.

14. Moreno M, Latorre A, Manzanares C, et al. Clinical management of tacrolimus drug interactions in renal transplant patients. Transplant Proc 1999;31:2252-3.

15. Kiuchi T, Tanaka K, Inomata Y, et al. Experience of tacrolimusbased immunosuppression in living-related liver transplantation complicated with graft tuberculosis: interaction with rifampicin and side effects. Transplant Proc 1996;28:3171-2.

16. Chenhsu RY, Loong CC, Chou MH, Lin MF, Yang WC. Renal allograft dysfunction associated with rifampin-tacrolimus interaction. Ann Pharmacother 2000;34:27-31.

17. Hebert MF, Fisher RM, Marsh CL, Dressler D, Bekersky I. Effects of rifampin on tacrolimus pharmacokinetics in healthy volunteers. J Clin Pharmacol 1999;39:91-6.

18. Furlan V, Perello L, Jacquemin E, Debray D, Taburet AM. Interactions between FK506 and rifampicin or erythromycin in pediatric liver recipients. Transplantation 1995;59:1217-18.

19. Undre NA. Pharmacokinetics of tacrolimus-based combination therapies. Nephrol Dial Transplant 2003;18(suppl 1):il2-15.

20. Hashimoto Y, Sasa H, Shimomura M, Inui K. Effects of intestinal and hepatic metabolism on the bioavailability of tacrolimus in rats. Pharm Res 1998;15:1609-13.

21. Undre NA, Stevenson P, Schafer A. Pharmacokinetics of tacrolimus: clinically relevant aspects. Transplant Proc 1999;31(suppl 7A):21S-4

22. Bekersky I, Dressler D, Mekki QA. Effect of low- and high-fat meals on tacrolimus absorption following $5 \mathrm{mg}$ single oral doses to healthy human subjects. J Clin Pharmacol 2001;41:176-82.

23. Bekersky I, Dressler D, Mekki Q. Effect of time of meal consumption on bioavailability of a single oral $5 \mathrm{mg}$ tacrolimus dose. J Clin Pharmacol 2001;41:289-97.

24. Maes BD, Lemahieu W, Kuypers D, et al. Differential effect of diarrhea on FK506 versus cyclosporine A trough levels and resultant prevention of allograft rejection in renal transplant recipients. Am J Transplant 2002;2:989-92.

25. Iglesias Berengue J, López-Espinosa JA, Ortega-López J, et al. Two- to three-fold increase in blood tacrolimus (FK506) levels during diarrhea in liver-transplanted children. Clin Transplant $2003 ; 17: 249-53$ 